\title{
Therapeutic Challenges for Knee Osteoarthritis
}

\author{
Farnad Imani ${ }^{1,{ }^{*}}$ and Vikram B. Patel ${ }^{2}$ \\ ${ }^{1}$ Pain Research Center, Iran University of Medical Sciences, Tehran, Iran \\ ${ }^{2}$ Phoenix Interventional Center for Advanced Learning, Algonquin, Illinois, USA \\ "Corresponding author: Pain Research Center, Iran University of Medical Sciences, Tehran, Iran. Email: farnadimani@yahoo.com
}

Received 2019 June 15; Accepted 2019 June 18.

Keywords: Knee, Osteoarthritis, Therapeutic

One of the most common degenerative joint diseases among the elderly who refer to pain clinics is knee osteoarthritis, the characteristics of which are progressive cartilage loss in the knee joint, synovial membrane changes, and reduced viscosity of the synovial fluid. It causes pain, joint mobility limitations, disability, and poor quality of life.

There is no specific, definite treatment, as yet, to reverse the destructive process involving joint inflammation for knee osteoarthritis, so the treatment is mostly symptomatic. Current consensus suggests that the knee osteoarthritis management should take a multidisciplinary approach involving conservative, surgical, as well as complementary therapies.

Physical therapy, oral medications, and intra-articular injections are considered as conservative treatment employed as the first step to treat mild to moderate osteoarthritis of the knee joint. The main goal of these modalities is to attenuate joint pain, improve joint function and quality of life, and slow down the progression of the disease. Several drugs, particularly nonsteroidal anti-inflammatory drugs (NSAIDs), corticosteroid, glucosamine, and hyaluronic acid, have been considered as nonsurgical pain management for osteoarthritis of the knee.

Hyaluronic acid is basically a natural glycosaminoglycan and a component of synovial fluid, which serves as a lubricant during joint movements. Intra-articular hyaluronic acid can restore the function of synovial fluid, protect against cartilage erosion, and alleviate synovial inflammation. It may also have some analgesic effect on the joints (1).

Injection of intra-articular corticosteroids has shown significant anti-inflammatory effect, and can boost the relative viscosity and concentration of hyaluronic acid in the arthritic knee. The duration of action of intra-articular corticosteroid injections has yet remained controversial, lying somewhere between 1 to 24 weeks, as suggested by different studies. However, there is an agreement that steroids start to relieve pain approximately one week after intra-articular injection. In addition, there are controversies concerning the intra-articular corticosteroid administration owing to the chondrolytic effects on cartilage and synovium (2).

Prolotherapy, also called "proliferation therapy," refers to an injection of hypertonic dextrose, a treatment option for a variety of painful chronic musculoskeletal pain conditions, particularly knee osteoarthritis. The main practical principle of prolotherapy is based on the injection of relatively small volumes of an irritant solution, generally hypertonic dextrose, at painful ligament and tendon attachments, as well as in adjacent joint spaces. In recent years, prolotherapy has been used for the treatment of knee osteoarthritis obstinate to other conservative options. Reports from articles have documented positive effects in knee osteoarthritis; the findings have not been synthesized, however (3).

An autologous concentration of human platelets by simple centrifugation of the patient's blood, which contains many components, including growth factors, cytokines, etc., is referred to as platelet-rich plasma (PRP) (4). It serves as a carrier for a large amount of growth factors, with the function of increasing tissue repair, which is progressively being used in the treatment of knee osteoarthritis (5). Compared to the physiologic plasma, PRP maintains four to six times the platelet concentration. According to some reports, platelet-rich plasma may cause precursor cells to migrate, proliferate, and differentiate in the synovial fat pad or cartilage. As a result, platelet-rich plasma could augment the repair of damaged cartilage, while reducing the pain and inflammation.

Several studies have compared platelet-rich plasma 
and hyaluronic acid to determine which was more effective, but did not reach an agreement with respect to pain relief and function recovery (6). In some studies, plateletrich plasma exhibited superior short-term results in relieving symptoms of mild to moderate knee osteoarthritis compared to hyaluronic acid (7). In other studies, however, no significant differences were observed between plateletrich plasma and hyaluronic acid in pain score at 6 or 12 months (8). Thus, it seems necessary to update the comparison between the two modalities.

The number of injections varies among studies (including a single or multiple injections) $(9,10)$. There was no difference between hyaluronic acid and single plateletrich plasma, while multiple platelet-rich plasma injections produced better results than the two groups.

Moreover, prior studies indicated that the efficacy of intra-articular injections of platelet-rich plasma or hyaluronic acid depended on the time interval after injection, and the improvement observed within two months might last up to six months of follow-up, but tended to diminish 6 to 12 months after the injection (11).

There are few complications with platelet-rich plasma, but they do increase if the solution is prepared with double centrifugation technique, as compared with the single method (12).

Adding some adjuvants, such as growth hormone and erythropoietin, to platelet rich plasma for intra-articular injection can improve function of the osteoarthritic knee in short time (13-16). Growth hormone is a prominent regulator of bone growth and mineral density; it also stimulates the cartilage growth probably by some mechanisms such as direct stimulation of cartilage cell proliferation. Healing of osteochondral defect can be accelerated by circulating growth hormone or one of its mediators (17).

Genicular nerve radiofrequency ablation has recently gained popularity as an intervention for refractory chronic knee pain (18). Long-term efficacy and side effects are still unknown. Thermal radiofrequency ablation for genicular nerves may cause vascular injury and osteonecrosis of the patella. Recently, cooled radiofrequency ablation for genicular nerves had appropriate results for knee osteoarthritis pain $(19,20)$. On the other hand, when cooled radiofrequency of the genicular nerves was performed a couple of weeks before elective total knee arthroplasty, there were no significant effects on postoperative analgesia or function in the early postoperative period (21). In pulsed radiofrequency, there is no tissue (nerve and vascular) damage. Pulsed radiofrequency of peripheral nerves and plexuses which supply the knee joint seemed to be a safe, efficient, and least invasive novel technique that addresses the peripheral (motor, sensory, and autonomic) nerves to provide prolonged relief of pain, stiffness, swelling, and the peripheral and central sensitivity in response to chronic pain in patients with knee osteoarthritis (22-24). Pulsed radiofrequency has been described in the treatment of chronic knee pain within the pericapsular nerve endings as well as sciatic and saphenous nerves (25-27). Furthermore, pulsed radiofrequency of the entire nerve supply of the knee showed improved functional outcome in patients with knee osteoarthritis (28).

In their study, Enteshari-Moghaddam and colleagues administered methotrexate to treat moderate to severe knee osteoarthritis. Their study showed no significant complications related to methotrexate administration; on the other hand, methotrexate was found effective in reducing pain and improving quality of life. Thus, it seemed an appropriate medication for moderate to severe knee osteoarthritis refractory to other conservative medications (29).

The treatment of knee osteoarthritis has advanced in recent years, in a distinctly separate manner, by products of regenerative medicine, including platelet-rich plasma, autologous conditioned serum, autologous protein solution, and mesenchymal stem cells (30). The aim is simply to reverse the swelling and conduct the joint to an anabolic, rather than catabolic, phase. This may enable the joint to heal itself and to eventual treatment, leading to pain relief, increased mobility, and potentially avoiding traumatic surgical operations. Further basic and clinical researches need to be conducted to investigate this hypothesis.

\section{Footnotes}

Conflict of Interests: It is not declared by the authors. Funding/Support: It is not declared by the authors.

\section{References}

1. He WW, Kuang MJ, Zhao J, Sun L, Lu B, Wang Y, et al. Efficacy and safety of intraarticular hyaluronic acid and corticosteroid for knee osteoarthritis: A meta-analysis. Int J Surg. 2017;39:95-103. doi: 10.1016/j.ijsu.2017.01.087. [PubMed: 28137554].

2. Juni P, Hari R, Rutjes AW, Fischer R, Silletta MG, Reichenbach $\mathrm{S}$, et al. Intra-articular corticosteroid for knee osteoarthritis. Cochrane Database Syst Rev. 2015;(10). CD005328. doi: 10.1002/14651858.CD005328.pub3. [PubMed: 26490760].

3. Rahimzadeh P, Imani F, Faiz SHR, Entezary SR, Zamanabadi MN, Alebouyeh MR. The effects of injecting intra-articular platelet-rich plasma or prolotherapy on pain score and function in knee osteoarthritis. Clin Interv Aging. 2018;13:73-9. doi: 10.2147/CIA.S147757. [PubMed: 29379278]. [PubMed Central: PMC5757490].

4. Foster TE, Puskas BL, Mandelbaum BR, Gerhardt MB, Rodeo SA. Platelet-rich plasma: From basic science to clinical applications. Am J Sports Med. 2009;37(11):2259-72. doi: 10.1177/0363546509349921. [PubMed: 19875361]. 
5. Patel S, Dhillon MS, Aggarwal S, Marwaha N, Jain A. Treatment with platelet-rich plasma is more effective than placebo for knee osteoarthritis: A prospective, double-blind, randomized trial Am J Sports Med. 2013;41(2):356-64. doi: 10.1177/0363546512471299. [PubMed: 23299850].

6. Andia I, Abate M. Knee osteoarthritis: Hyaluronic acid, platelet-rich plasma or both in association? Expert Opin Biol Ther. 2014;14(5):63549. doi: 10.1517/14712598.2014.889677. [PubMed: 24533435].

7. Sanchez M, Fiz N, Azofra J, Usabiaga J, Aduriz Recalde E, Garcia Gutierrez A, et al. A randomized clinical trial evaluating plasma rich in growth factors (PRGF-Endoret) versus hyaluronic acid in the short-term treatment of symptomatic knee osteoarthritis. Arthroscopy. 2012;28(8):1070-8. doi: 10.1016/j.arthro.2012.05.011. [PubMed: 22840987].

8. Cole BJ, Karas V, Hussey K, Pilz K, Fortier LA. Hyaluronic acid versus platelet-rich plasma: A prospective, double-blind randomized controlled trial comparing clinical outcomes and effects on intraarticular biology for the treatment of knee osteoarthritis. Am J Sports Med. 2017;45(2):339-46. doi: 10.1177/0363546516665809. [PubMed 28146403].

9. Raeissadat SA, Rayegani SM, Hassanabadi H, Fathi M, Ghorbani E, Babaee M, et al. Knee osteoarthritis injection choices: Platelet- rich plasma (PRP) versus hyaluronic acid (a one-year randomized clinical trial). Clin Med Insights Arthritis Musculoskelet Disord. 2015;8:1-8. doi: 10.4137/CMAMD.S17894. [PubMed: 25624776]. [PubMed Central: PMC4287055].

10. Duymus TM, Mutlu S, Dernek B, Komur B, Aydogmus S, Kesiktas FN. Choice of intra-articular injection in treatment of knee osteoarthritis: Platelet-rich plasma, hyaluronic acid or ozone options. Knee Surg Sports Traumatol Arthrosc. 2017;25(2):485-92. doi: 10.1007/s00167-0164110-5. [PubMed: 27056686].

11. Filardo G, Kon E, Buda R, Timoncini A, Di Martino A, Cenacchi A, et al. Platelet-rich plasma intra-articular knee injections for the treatment of degenerative cartilage lesions and osteoarthritis. Knee Surg Sports Traumatol Arthrosc. 2011;19(4):528-35. doi: 10.1007/s00167-0101238-6. [PubMed: 20740273].

12. Frizziero A, Giannotti E, Oliva F, Masiero S, Maffulli N. Autologous conditioned serum for the treatment of osteoarthritis and other possible applications in musculoskeletal disorders. Br Med Bull. 2013;105:16984. doi: 10.1093/bmb/lds016. [PubMed: 22763153].

13. Rahimzadeh P, Imani F, Faiz SH, Alebouyeh MR, Azad-Ehyaei D, Bahari L, et al. Adding intra-articular growth hormone to platelet rich plasma under ultrasound guidance in knee osteoarthritis: A comparative double-blind clinical trial. Anesth Pain Med. 2016;6(6). e41719. doi: 10.5812/aapm.41719. [PubMed: 28975078]. [PubMed Central: PMC5560632].

14. Derakhshan P. What is the role of erythropoietin prolotherapy on pain relief of knee osteoarthritis? J Res Med Sci. 2018;23:50. doi: 10.4103/jrms.JRMS_802_16. [PubMed: 30057634]. [PubMed Central: PMC6040149].

15. Rahimzadeh P, Imani F, Faiz SH, Entezary SR, Nasiri AA, Ziaeefard M. Investigation the efficacy of intra-articular prolotherapy with erythropoietin and dextrose and intra-articular pulsed radiofrequency on pain level reduction and range of motion improvement in primary osteoarthritis of knee. J Res Med Sci. 2014;19(8):696-702. [PubMed 25422652]. [PubMed Central: PMC4235087].

16. Imani F, Entezary S, Razi M, Jafarian AA, Yousefshahi F, Etemadi $\mathrm{H}$, et al. The effect of intra-articular meperidine and bupivacaine $0.5 \%$ on postoperative pain of arthroscopic knee surgery; a random- ized double blind clinical trial. Anesth Pain Med. 2015;5(1). e27470. doi: 10.5812/aapm.27470. [PubMed: 25830119]. [PubMed Central: PMC4363738]

17. Bail H, Klein P, Kolbeck S, Krummrey G, Weiler A, Schmidmaier G, et al. Systemic application of growth hormone enhances the early healing phase of osteochondral defects-a preliminary study in micropigs. Bone. 2003;32(5):457-67. [PubMed: 12753861].

18. Kim SY, Le PU, Kosharskyy B, Kaye AD, Shaparin N, Downie SA. Is genicular nerve radiofrequency ablation safe? A literature review and anatomical study. Pain Physician. 2016;19(5):E697-705. [PubMed: 27389113].

19. Reddy RD, McCormick ZL, Marshall B, Mattie R, Walega DR. Cooled radiofrequency ablation of genicular nerves for knee osteoarthritis pain: A protocol for patient selection and case series. Anesth Pain Med. 2016;6(6). e39696. doi: 10.5812/aapm.39696. [PubMed: 28975074]. [PubMed Central: PMC5560582].

20. Davis T, Loudermilk E, DePalma M, Hunter C, Lindley DA, Patel N, et al. Twelve-month analgesia and rescue, by cooled radiofrequency ablation treatment of osteoarthritic knee pain: Results from a prospective, multicenter, randomized, cross-over trial. Reg Anesth Pain Med. 2019. doi: 10.1136/rapm-2018-100051. [PubMed: 30772821].

21. Walega D, McCormick Z, Manning D, Avram M. Radiofrequency ablation of genicular nerves prior to total knee replacement has no effect on postoperative pain outcomes: A prospective randomized shamcontrolled trial with 6-month follow-up. Reg Anesth Pain Med. 2019. doi: 10.1136/rapm-2018-100094. [PubMed: 31023931].

22. Sluijter ME, Imani F. Evolution and mode of action of pulsed radiofrequency. Anesth Pain Med. 2013;2(4):139-41. doi: 10.5812/aapm.10213. [PubMed: 24223349]. [PubMed Central: PMC3821144].

23. Imani F, Gharaei H, Rezvani M. Pulsed radiofrequency of lumbar dorsal root ganglion for chronic postamputation phantom pain. Anesth Pain Med. 2012;1(3):194-7. doi: 10.5812/kowsar.22287523.3768. [PubMed: 24904793]. [PubMed Central: PMC4018701].

24. Imani F. Using pulsed radiofrequency for chronic pain. Anesth Pain Med. 2012;1(3):155-6. doi: 10.5812/kowsar.22287523.4047. [PubMed: 24904784]. [PubMed Central: PMC4018683].

25. Masala S, Fiori R, Raguso M, Morini M, Calabria E, Simonetti G. Pulsedose radiofrequency for knee osteoartrithis. Cardiovasc Intervent Radiol. 2014;37(2):482-7. doi: 10.1007/s00270-013-0694-z. [PubMed: 23942592].

26. Fucci Djibilian ER, Pascual-Ramirez J, Martinez-Marcos A, Mantecon JM. Ultrasound-guided sciatic nerve pulsed radiofrequency for chronic knee pain treatment: A novel approach. J Anesth. 2013;27(6):935-8. doi: 10.1007/s00540-013-1624-6. [PubMed: 23978947].

27. Akbas M, Luleci N, Dere K, Luleci E, Ozdemir U, Toman H. Efficacy of pulsed radiofrequency treatment on the saphenous nerve in patients with chronic knee pain. J Back Musculoskelet Rehabil. 2011;24(2):77-82. doi: 10.3233/BMR20110277. [PubMed: 21558611].

28. Vas L, Pai R, Khandagale N, Pattnaik M. Pulsed radiofrequency of the composite nerve supply to the knee joint as a new technique for relieving osteoarthritic pain: A preliminary report. Pain Physician. 2014;17(6):493-506. [PubMed: 25415774].

29. Enteshary-Moghaddam A, Isazadehfar K, Habibzadeh A, Hemmati M. Efficacy of methotrexate on pain severity reduction and improvement of quality of life in patients with moderate to severe knee osteoarthritis. Anesthesiol Pain Med. 2019;9(3). doi:10.5812/aapm.89990.

30. Zhao L, Kaye AD, Abd-Elsayed A. Stem cells for the treatment of knee osteoarthritis: A comprehensive review. Pain Physician. 2018;21(3):229-42. [PubMed: 29871367]. 